\title{
Effect of type 2 diabetes on mortality risk associated with end-stage kidney disease
}

\author{
E. Villar • K. R. Polkinghorne • S. H. Chang • \\ S. J. Chadban • S. P. McDonald
}

Received: 15 June 2009/Accepted: 6 August 2009 /Published online: 9 September 2009

(C) Springer-Verlag 2009

\begin{abstract}
Aims/hypothesis Patients with end-stage kidney disease (ESKD) and patients with diabetes mellitus experience higher mortality rates than the general population. Whether ESKD imparts the same excess in mortality risk for those with diabetes as it does for those without diabetes is unknown.

Methods Included in the study were all white patients aged $\geq 25$ years with incident ESKD and type 2 diabetes $(n=4,141)$ or with incident ESKD but without diabetes $(n=13,289)$ in Australia from 1991 to 2005 , and all the individuals aged $\geq 25$ years without ESKD and with type 2 diabetes $(n=909)$ or without ESKD without diabetes $(n=10,302)$ enrolled in the AusDiab Study - a nationwide Australian representative cohort-from 1999 to 2005. Excess mortality was analysed in patients with ESKD by diabetes status, using age-, sex- and diabetes-status-specific standardised mortality ratios (SMRs) in the first 8 years after
\end{abstract}

E. Villar · K. R. Polkinghorne $\cdot$ S. H. Chang $\cdot$ S. J. Chadban ·

S. P. McDonald

Australia and New Zealand Dialysis and Transplant Registry,

Woodville, South Australia, Australia

E. Villar $(\bowtie)$

Service de Néphrologie-Transplantation Rénale,

Hospices Civils de Lyon, Centre Hospitalier Lyon Sud, 165,

Chemin du Grand Revoyet,

69495 Pierre Bénite Cedex, France

e-mail: emmanuel.villar@chu-lyon.fr

K. R. Polkinghorne

Department of Nephrology, Monash Medical Centre,

Melbourne, Victoria, Australia

K. R. Polkinghorne

Monash University,

Melbourne, Australia first renal replacement therapy among ANZDATA patients relative to AusDiab participants.

Results The SMRs in patients with ESKD were, in nondiabetic patients and in those with type 2 diabetes, respectively: $14.2(95 \%$ CI $13.9-14.6)$ and $10.8(95 \%$ CI 10.4-11.2) $(p<0.01)$; in people aged $<60$ years, $28.7(95 \%$ CI 27.2-30.4) and $18.6(95 \%$ CI 17.1-20.4) $(p<0.01)$; in people aged $\geq 60$ years, $12.5(95 \%$ CI $12.1-12.9)$ vs $9.7(95 \%$ CI 9.3-10.1) $(p<0.01)$; in men, 11.0 (95\% CI 10.7-11.4) vs 8.9 (95\% CI 8.4-9.3) $(p<0.01)$; and in women, $23.4(95 \%$ CI 22.5-24.3) vs 16.2 (95\% CI 15.2-17.3) $(p<0.01)$.

Conclusions/interpretation ESKD was associated with a greater relative increase in mortality in the non-diabetic study populations than in the type 2 diabetes population. Excess mortality was greater among younger people and women.

Keywords ANZDATA Registry · AusDiab Study - Diabetes · End-stage kidney disease $\cdot$ Standardised mortality ratio

S. H. Chang $\cdot$ S. P. McDonald

Department of Nephrology,

The Queen Elizabeth Hospital,

Woodville, South Australia, Australia

S. H. Chang $\cdot$ S. P. McDonald

University of Adelaide,

Adelaide, South Australia, Australia

S. J. Chadban

Renal Medicine and Transplantation,

Royal Prince Alfred Hospital,

Camperdown, New South Wales,

Australia

S. J. Chadban

University of Sydney,

Sydney, New South Wales,

Australia 


$\begin{array}{ll}\text { Abbreviations } \\ \text { AusDiab } & \begin{array}{l}\text { Australian Diabetes, Obesity and Lifestyle } \\ \text { Study }\end{array} \\ \text { ANZDATA } & \begin{array}{l}\text { Australian and New Zealand Dialysis and } \\ \text { Transplant Registry }\end{array} \\ \text { CKD } & \text { Chronic kidney disease } \\ \text { CVD } & \text { Cardiovascular disease } \\ \text { eGFR } & \text { Estimated GFR } \\ \text { ESKD } & \text { End-stage kidney disease } \\ \text { RRT } & \text { Renal replacement therapy } \\ \text { SMR } & \text { Standardised mortality ratio } \\ \text { uPCR } & \text { Urine protein/creatinine ratio }\end{array}$

\section{Introduction}

End-stage kidney disease (ESKD) is associated with an excess mortality compared with the age- and sex-matched general population [1]. An analysis of French patients with incident ESKD reported a standardised mortality ratio (SMR) for that group compared with the general population [2]. In that study, the excess mortality associated with ESKD was higher in patients with diabetic nephropathy than in those with other causes of kidney failure [2]. However, whether ESKD imparts the same relative mortality risk among those with diabetes as it does for those without diabetes is unknown, because that study did not take into account the diabetic status of the non-ESKD reference population [2].

In Australia, the availability of a national treated ESKD Registry (ANZDATA Registry) [3] and mortality rates from a large, population-based cohort study (Australian Diabetes, Obesity and Lifestyle [AusDiab] Study) [4], allowed us to examine mortality rates for Australian patients with ESKD by the presence or absence of type 2 diabetes, and calculate age-, sex- and diabetes-specific SMRs.

\section{Methods}

The study was approved by the ANZDATA review board and was carried out in accordance with the Declaration of Helsinki (revised 2000) of the World Medical Association.

Mortality rates in the Australian population The AusDiab Study is a nationally representative cohort study of 11,247 non-institutionalised adults aged $>25$ years conducted in 1999-2000 in Australia [4]. Participants were predominantly white $(92.9 \%)$. The proportions of participants with type 1 diabetes, type 2 diabetes and without diabetes were $0.3 \%$, $8.1 \%$ and $91.6 \%$, respectively. The 5 year follow-up of AusDiab participants provided population-based 1 year mortality rates in Australia by age band, sex and diabetic status [4].

Australian ESKD patients Data from the ANZDATA Registry were used to calculate mortality rates in Australian patients with incident ESKD [3]. We restricted the ESKD population to all white patients aged $>25$ years who began chronic dialysis in Australia from 1 April 1991 to 31 December 2005. Patients with type 1 diabetes and ESKD were excluded as the number in the AusDiab population was too small to calculate a mortality rate. Demographic, clinical data and comorbid conditions were prospectively collected at the start of dialysis [3]. Patients were followed until they died or 31 December 2005 [3].

Standardised mortality ratio Age-, sex- and diabetesstatus-specific SMR with $95 \%$ confidence intervals among ESKD groups were computed using standard methods [5]. SMRs were computed for type 2 diabetic and non-diabetic ESKD patients against corresponding non-ESKD AusDiab groups. To ensure sufficient statistical power, we limited the analysis to the first 8 years of replacement renal therapy (RRT) ( $>50$ remaining patients in all ESKD patient subgroups for each year).

In the ESKD patient groups, we observed the number of deaths $\left(\mathrm{O}_{\text {Deaths }}\right)$ annually from the first $\mathrm{RRT}$, conditional on survival to that year. The expected number of deaths $\left(E_{\text {Deaths }}\right)$ was derived from the 1 year mortality rates from the AusDiab Study, matched for age band (25-34, 35-44, $45-54,55-64,65-74,75-84$, and $\geq 85$ years), sex and diabetic status.

$E_{\text {Deaths }}$ was the sum of expected numbers of deaths for each stratum of interest. The SMR was the ratio of $\mathrm{O}_{\text {Deaths }}$ to $E_{\text {Deaths }}[5]$.

When the SMR heterogeneity test [5] over years after first RRT did not reach statistical significance, we presented a single SMR for the 8 year period. Otherwise, we presented the SMR separately for each year.

Comparison of SMR between patient subgroups was performed with the $\chi^{2}$ test [5], stratified by age band and sex, and computed annually from first RRT using the Mantel-Haenszel method.

All statistical analyses were performed with S-PLUS 6.0 Software Professional Release 2 (Insightful, Seattle, WA, USA). $p$ values $<0.05$ were considered statistically significant.

\section{Results}

Characteristics of type 2 diabetic and non-diabetic ESKD and AusDiab populations Participants with type 2 diabetes and ESKD were older, and more likely to be male and to 
Table 1 Baseline characteristics and RRT in type 2 diabetic and non-diabetic Australian patients with ESKD (1991-2005)

\begin{tabular}{|c|c|c|c|}
\hline Characteristic & With type 2 diabetes $(n=4,141,23.8 \%)$ & Without diabetes $(n=13,289,76.2 \%)$ & $p$ value \\
\hline Men, $n(\%)$ & $2,606(62.9)$ & $7,841(59.0)$ & $<0.0001$ \\
\hline Age at first RRT (years) (mean \pm SEM) & $65.5 \pm 9.9$ & $60.0 \pm 15.0$ & $<0.0001$ \\
\hline Age at first RRT (years) (median) & 66.7 & 62.7 & \\
\hline Primary renal disease, $n(\%)$ & & & - \\
\hline Diabetes & $2,627(63.4)$ & - & \\
\hline Renal vascular disease & $438(10.6)$ & $2,290(17.2)$ & \\
\hline $\mathrm{GN}$ and related disease & $461(11.1)$ & $5,043(38.0)$ & \\
\hline Polycystic & $76(1.8)$ & $1,432(10.8)$ & \\
\hline Other & $539(13.0)$ & $4,524(34.0)$ & \\
\hline \multicolumn{4}{|l|}{ Co-morbidity at first RRT, $n(\%)$} \\
\hline Chronic lung disease & $795(19.2)$ & $2,138(16.1)$ & $<0.0001$ \\
\hline Coronary artery disease & $2,609(63.0)$ & $4,514(34.0)$ & $<0.0001$ \\
\hline Peripheral vascular disease & $2,028(49.0)$ & 2,477 (18.6) & $<0.0001$ \\
\hline Cerebrovascular disease & $973(23.5)$ & $1,708(12.9)$ & $<0.0001$ \\
\hline 90 day RRT modality, $n(\%)$ & & & $<0.0001$ \\
\hline Haemodialysis & $2,636(56.4)$ & $8,162(61.4)$ & \\
\hline Peritoneal dialysis & $1,487(35.9)$ & $4,642(34.9)$ & \\
\hline Renal transplantation & $18(0.4)$ & $485(3.7)$ & \\
\hline Renal transplant, $n(\%)^{\mathrm{a}}$ & $205(5.0)$ & $3,604(27.1)$ & $<0.0001$ \\
\hline Median time to transplant (months) $(95 \% \mathrm{CI})$ & $19.9(16.7-24.4)$ & $18.9(17.9-19.9)$ & \\
\hline Crude median survival (months) $(95 \% \mathrm{CI})$ & $37.4(35.6-38.8)$ & $70.5(68.0-72.8)$ & $<0.0001$ \\
\hline
\end{tabular}

Data are presented as mean $\pm \mathrm{SEM}, n(\%)$, median or median $(95 \% \mathrm{CI})$

${ }^{a}$ Analyses restricted to patients younger than 70 years, including living donor and deceased donor renal transplantation

GN, glomerulonephritis; RRT, renal replacement therapy

have comorbid conditions than the non-diabetic patients with ESKD (Table 1). The overall crude 1 year mortality rate was twofold higher in type 2 diabetic than in nondiabetic patients with ESKD (Table 2).
In the reference population (AusDiab), the mean estimated GFRs (eGFRs) were over $60 \mathrm{ml} \mathrm{min}{ }^{-1} 1.73 \mathrm{~m}^{-2}$ and mean urine protein/creatinine ratios (uPCRs) were within the normal range $(<249 \mathrm{mg} / \mathrm{mmol})$ in both groups

Table 2 One year mortality rates and 95\% CI by age, sex and diabetic status in Australian patients with ESKD (ANZDATA Registry, 1991-2005)

\begin{tabular}{llll}
\hline & & 1 year death rate per 1,000 person-years & \\
\cline { 2 - 3 } Age band (years) & Sex & With type 2 diabetes $(n=4,141,23.8 \%)$ & Without diabetes $(n=13,289,76.2 \%)$ \\
\hline $25-54$ & Male & $129.609(111.267-150.111)$ & $32.075(24.100-41.431)$ \\
$25-54$ & Female & $122.137(99.931-149.150)$ & $35.848(32.311-39.666)$ \\
$55-64$ & Male & $182.690(165.673-200.980)$ & $112.137(87.255-140.437)$ \\
$55-64$ & Female & $198.054(174.924-223.392)$ & $115.911(89.706-146.179)$ \\
$65-74$ & Male & $253.537(192.907-323.246)$ & $209.039(175.813-245.729)$ \\
$65-74$ & Female & $304.205(277.008-333.349)$ & $208.331(171.153-250.331)$ \\
$75-84$ & Male & $327.395(288.515-370.055)$ & $304.020(243.547-371.837)$ \\
$75-84$ & Female & $362.970(307.208-425.927)$ & $306.872(227.229-402.428)$ \\
$\geq 85$ & Male & $587.919(281.930-1081.20)$ & $506.664(378.400-664.422)$ \\
$\geq 85$ & Female & $495.770(160.975-1156.96)$ & $424.189(279.543-617.173)$ \\
Overall & - & $224.031(196.907-253.846)$ & $111.352(102.888-120.327)$ \\
\hline
\end{tabular}

Data are presented as rate $(95 \% \mathrm{CI})$

$* * * p<0.0001$ vs without diabetes 
Table 3 Baseline characteristics of the AusDiab population (AusDiab Study, 1999-2005) [4]

\begin{tabular}{|c|c|c|c|}
\hline Characteristic & With type 2 diabetes $(n=908)$ & Without diabetes $(n=10,302)$ & $p$ value \\
\hline Age (years) & $63.2 \pm 12.3$ & $50.8 \pm 14.2$ & $<0.0001$ \\
\hline Men & $477(52.5)$ & $4,551(44.2)$ & $<0.0001$ \\
\hline History of cardiovascular disease $\mathrm{a}^{\mathrm{a}}$ & $157(17.3)$ & $582(5.6)$ & $<0.0001$ \\
\hline $\mathrm{eGFR}^{\mathrm{b}}\left(\mathrm{ml} \mathrm{min}-1.73 \mathrm{~m}^{-2}\right)$ & $71.9 \pm 14.7$ & $76.3 \pm 12.6$ & $<0.0001$ \\
\hline uPCR (mg/mmol) & $113 \pm 260$ & $51 \pm 136$ & $<0.0001$ \\
\hline
\end{tabular}

${ }^{a}$ Self-reported history of stroke, heart attack or angina

${ }^{\mathrm{b}}$ Modification of Diet in Renal Disease (MDRD) equation

Data are presented as mean \pm SD or $n(\%)$

(Table 3). Type 2 diabetic patients were older, more likely to be male and to have a history of cardiovascular disease (CVD) than those without diabetes, and they had lower mean eGFRs and higher uPCRs than non-diabetic participants (Table 3 ). The overall crude 1 year mortality rate was fivefold higher in type 2 diabetic AusDiab participants than in non-diabetic AusDiab participants (Table 4).

Excess mortality associated with ESKD After adjusting for age and sex, the SMR was significantly higher in nondiabetic than in type 2 diabetic patients with ESKD: 14.2 (95\% CI 13.9-14.6) compared with 10.8 (95\% CI 10.4-11.2), respectively $(p<0.01)$. When the analysis was restricted to type 2 diabetic patients with diabetic nephropathy $(n=2,627$, $63.4 \%$ ), the SMR was 11.5 (95\% CI 10.9-12.1) (not significantly different from the SMR in all type 2 diabetic individuals).

Impact of age and sex on excess mortality The SMRs in the non-diabetic group with ESKD vs those with type 2 diabetes and ESKD were: in patients aged $<60$ years, $28.7(95 \%$ CI 27.2-30.4) vs 18.6 (95\% CI 17.1-20.4), respectively, $(p<0.01)$; in participants aged $\geq 60$ years, $12.5(95 \% \mathrm{CI}$
12.1-12.9) vs 9.7 (95\% CI 9.3-10.1), respectively $(p<0.01)$; in men, 11.0 (95\% CI 10.7-11.4) vs 8.9 (95\% CI 8.4-9.3) $(p<0.01)$; and in women, 23.4 (95\% CI 22.5-24.3) vs 16.2 (95\% CI 15.2-17.3) $(p<0.01)$. In both groups, the SMRs were higher in patients aged $<60$ years than in patients aged $\geq 60$ years $(p<0.01)$ and in women than in men $(p<0.01)$. When the analyses were restricted to type 2 diabetic patients with diabetic nephropathy, the results were not significantly changed (data not shown).

\section{Discussion}

The excess relative mortality associated with ESKD was greater in non-diabetic individuals (mortality risk increased $\times 14$ ) than in those with type 2 diabetes (risk $\times 10$ ) when compared with the non-ESKD population with the same diabetes status, although patients with type 2 diabetes and ESKD had a mean absolute overall mortality twofold higher than that of non-diabetic patients with $\operatorname{ESKD}[6,7]$. Restricting the analyses to type 2 diabetes patients with diabetic nephropathy did not change the results.
Table 4 One year mortality rates and $95 \%$ CI by age, sex and diabetic status (AusDiab Study, 1999-2005) [4]
Data are presented as rate (95\% CI)

${ }^{a}$ No deaths occurred in this group

$* * * p<0.0001$ vs without diabetes

\begin{tabular}{llcc}
\hline & & 1 year death rate per 1,000 person-years \\
\cline { 3 - 4 } Age band & Sex & With type 2 diabetes $(n=908)$ & Without diabetes $(n=10,302)$ \\
\hline $25-54$ & Male & $3.892(0.471-14.058)$ & $1.192(0.681-1.935)$ \\
$25-54$ & Female & $0.000(-)^{\mathrm{a}}$ & $0.747(0.398-1.278)$ \\
$55-64$ & Male & $14.644(6.696-27.799)$ & $3.114(1.743-5.137)$ \\
$55-64$ & Female & $2.089(0.052-11.640)$ & $1.200(0.483-2.474)$ \\
$65-74$ & Male & $24.022(13.731-39.011)$ & $11.396(8.024-15.709)$ \\
$65-74$ & Female & $17.693(8.832-31.659)$ & $5.875(3.725-8.817)$ \\
$75-84$ & Male & $38.314(22.707-60.553)$ & $34.821(25.860-43.784)$ \\
$75-84$ & Female & $29.779(17.347-47.679)$ & $16.690(11.690-23.107)$ \\
$\geq 85$ & Male & $135.911(67.846-243.183)$ & $84.455(53.537-126.725)$ \\
$\geq 85$ & Female & $100.341(43.320-197.713)$ & $72.841(47.582-106.730)$ \\
Overall & - & $20.576(16.607-25.207)$ & $4.870(4.296-5.500)$ \\
\hline
\end{tabular}


The difference in the relative impact of ESKD on individuals with and without type 2 diabetes was not accounted for by differences in the age and sex structures of the groups. However, there are a number of possible explanations. People who develop ESKD have survived a period of chronic kidney disease and other competing risks of death-if these risks were higher among type 2 diabetic than non-diabetic patients, a bias may be introduced. Differences in the prevalence of CVD and the associated mortality between patients with ESKD and the general population by diabetes status are likely to exist: in the US Medicare population aged $\geq 65$ years, the prevalence of peripheral vascular disease was 3.4-fold higher in nondiabetic patients with chronic kidney disease (CKD) than in those without CKD (32.0\% vs $9.6 \%$ ) but was only 2.1 -fold higher in diabetic patients with CKD than in those without CKD (38.6\% vs $18.0 \%)$ [8]. This may confound the diabetes association. Unfortunately, differences in CVD recording methods between the studied cohorts did not allow stratifying analyses by CVD status $[3,4]$.

The relative mortality among ESKD groups decreased with increasing age, regardless of diabetic status. This is explained in part by very low mortality rates in the younger population without ESKD, leading to a larger 'health gap' between populations with and without ESKD in younger compared with older participants [2]. The SMRs were higher in women as well: mortality rates were similar between the sexes in those with ESKD, but lower in women without ESKD - that is, the female survival advantage seen in the general population was lost in ESKD patients [2].

Our study has limitations. In the AusDiab population, the overall mortality rate was 6.1 per 1,000 person-years [4]. We assumed that the 1 year mortality rates from the AusDiab Study were stable over the 1999-2005 period and applicable to the 1991-2005 period. However, national death rates from the Australian Bureau of Statistics for the Australian population aged $>25$ years were nine to ten per 1,000 person-years between 1991 and 2005 [9]. This gap reflects the inclusion of a variety of groups in the population-based figures that were excluded from the AusDiab Study. Institutionalised individuals and indigenous people were not included when the AusDiab population was constituted [4]. However, the use of SMR based on a nationally representative cohort without the studied disease may be more accurate than one based on the general population, which will include people with a variety of other chronic diseases leading to an underestimation of excess mortality due to a specific disease [10]. Some bias may remain - the AusDiab population included $7.1 \%$ of non-white participants and we were not able to exclude them from this cohort. We excluded the one-quarter of patients with incident ESKD in Australia who were nonwhite [6]. Although an imbalance in type 2 diabetes prevalence between cohorts by race will introduce bias, this will be limited by the small proportion of non-white members of the AusDiab cohort. At least, the SMR method assessed excess mortality adjusted for a limited number of mortality risks (usually, age and sex) [5]. In our study, comparisons between subgroups should take into account that SMRs were adjusted only for age, sex and diabetic status.

In conclusion, patients with type 2 diabetes and ESKD have an overall higher absolute mortality risk but, when comparing patients with ESKD with a reference non-ESKD population with the corresponding diabetes status, ESKD was associated with a greater excess mortality in nondiabetic individuals than in those with type 2 diabetes. This effect varied with age and sex and may be due to differential cardiovascular risk gaps between patients with and without ESKD in type 2 diabetic and non-diabetic populations. These results underline that the risk for death was increased more than tenfold for the population reaching ESKD.

Acknowledgements We gratefully acknowledge all ANZDATA Registry participants, especially the nephrologists and professionals who collected the data and conducted the quality control. We gratefully acknowledge all the AusDiab support staff, and especially the participants for volunteering their time to be involved in the study. The interpretation of reported data is the responsibility of the authors and in no way should be seen as an official interpretation of the ANZDATA Registry and of the AusDiab Study support staff. E. Villar and S. McDonald have full access to all data used in this study. No author has access to personally identifying information.

Financial disclosures The ANZDATA Registry is funded by the Australian Government Department of Health of Ageing, the New Zealand Ministry of Health and by Kidney Health Australia. The Registry has also received contributions from various pharmaceutical and dialysis companies on an unrestricted basis. The AusDiab Study is supported by an NHMRC project grant (233200) and in-kind support from the Australian Institute of Health and Welfare, the Australian Government Department of Health and Ageing. The Registry has also received contributions from various pharmaceutical and dialysis companies on an unrestricted basis. E. Villar was supported by unconditional research grants from the Hospices Civils de Lyon (France), and from Novartis and Roche pharmaceutical companies (France). The sponsors have not been involved in any way in the study design, data interpretation or manuscript editing.

Duality of interest The authors declare that there is no duality of interest associated with this manuscript.

\section{References}

1. Levey AS, Beto JA, Coronado BE et al (1998) Controlling the epidemic of cardiovascular disease in chronic renal disease: what do we know? What do we need to learn? Where do we go from here? National Kidney Foundation Task Force on Cardiovascular Disease. Am J Kidney Dis 32:853-906 
2. Villar E, Remontet L, Labeeuw M, Ecochard R (2007) Effects of age, sex and diabetes on excess death in end-stage renal failure. J Am Soc Nephrol 18:2125-2134

3. ANZDATA Registry report 2006 (2007) Australia and New Zealand Dialysis and Transplant Registry. ANZDATA Registry, Adelaide

4. Barr ELM, Magliano DJ, Zimmet PZ, the AusDiab Steering Committee et al (2005) The Australian Diabetes, Obesity and Lifestyle Study. Tracking the accelerating epidemic: its cause and outcome. The International Diabetes Institute, Melbourne

5. Breslow NE, Day NA (1987) Statistical methods in cancer research: volume II-the design and analysis of cohort studies (IARC scientific publications no. 82). International Agency for Research on Cancer, Lyon

6. Villar E, Chang SH, McDonald SP (2007) Incidences, treatments, outcomes, and gender effect on survival in end-stage renal disease patients by diabetic status in Australia and New Zealand (19912005). Diabetes Care 30:3070-3076

7. Karamé A, Labeeuw M, Trolliet P et al (2009) The impact of type 2 diabetes on mortality in end-stage renal disease patients differs between genders. Nephron Clin Pract 112:268-275

8. Foley RN, Murray AM, Li S et al (2005) Chronic kidney disease and the risk for cardiovascular disease, renal replacement, and death in the United States Medicare population, 1998 to 1999. J Am Soc Nephrol 16:489-495

9. Australian Bureau of Statistics (2009) Deaths, Australia, 2007 (cat. no. 3302.0). Available from www.abs.gov.au/AUSSTATS/ abs@.nsf/Lookup/3302.0Main+Features12007?OpenDocument, accessed 14 June 2009

10. Jones ME, Swerdlow AJ (1998) Bias in the standardized mortality ratio when using the general population rates to estimate expected number of deaths. Am J Epidemiol 148:1012-1017 